\section{(C) OPEN ACCESS}

\title{
The Test Your Memory for Mild Cognitive Impairment (TYM-MCI)
}

\author{
Jeremy M Brown, ${ }^{1,2}$ Claire J Lansdall, ${ }^{3}$ Julie Wiggins, ${ }^{3}$ Kate E Dawson, ${ }^{3}$ \\ Kristina Hunter, ${ }^{1}$ James B Rowe, ${ }^{1,3}$ Richard A Parker ${ }^{4}$
}

- Additional material is published online only. To view, please visit the journal online (http://dx.doi.org/10.1136/ jnnp-2016-315327)

${ }^{1}$ Department of Neurology, Cambridge University Hospitals, Hills Road, Cambridge, UK ${ }^{2}$ Queen Elizabeth Hospital NHS Trust, Gayton Road, King's Lynn, Norfolk, UK

${ }^{3}$ Cambridge University Department of Clinica Neurosciences, Cambridge, UK ${ }^{4}$ Edinburgh Clinical Trials Unit, Usher Institute of Population Health Sciences and Informatics, University of Edinburgh, Edinburgh, UK

Correspondence to Dr Jeremy M Brown, Department of Neurology, Queen Elizabeth Hospital King's Lynn NHS Trust, King's Lynn, UK; jmb75@medschl.cam.ac.uk

JBR and RAP contributed equally.

Received 21 November 2016 Revised 2 May 2017 Accepted 8 May 2017

Published Online First 14 September 2017

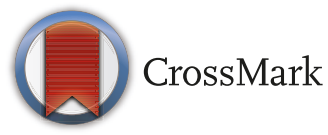

To cite: Brown JM, Lansdall CJ, Wiggins J, et al. J Neurol Neurosurg Psychiatry 2017:88:1045-1051.

\section{ABSTRACT}

Background To validate a short cognitive test: the Test Your Memory for Mild Cognitive Impairment (TYM-MCI) in the diagnosis of patients with amnestic mild cognitive impairment or mild Alzheimer's disease (aMCI/AD).

Methods Two hundred and two patients with mild memory problems were recruited. All had 'passed' the Mini-Mental State Examination (MMSE). Patients completed the TYM-MCl, the Test Your Memory test (TYM), MMSE and revised Addenbrooke's Cognitive Examination (ACE-R), had a neurological examination, clinical diagnostics and multidisciplinary team review. Results As a single test, the TYM-MCI performed as well as the ACE-R in the distinction of patients with aMCI/ $A D$ from patients with subjective memory impairment with a sensitivity of 0.79 and specificity of 0.91 . Used in combination with the ACE-R, it provided additional value and identified almost all cases of aMCI/AD. The TYM-MCI correctly classified most patients who had equivocal ACE-R scores. Integrated discriminant improvement analysis showed that the TYM-MCl added value to the conventional memory assessment. Patients initially diagnosed as unknown or with subjective memory impairment who were later rediagnosed with aMCI/AD scored poorly on their original TYM-MCl.

Conclusion The TYM-MCl is a powerful short cognitive test that examines verbal and visual recall and is a valuable addition to the assessment of patients with aMCI/AD. It is simple and cheap to administer and requires minimal staff time and training.

\section{INTRODUCTION}

Early accurate diagnosis of Alzheimer's disease (AD) is a major global health priority. ${ }^{1}$ Early diagnosis is important for patients and helps plan care. Emerging disease-modifying treatments for $\mathrm{AD}$ are likely to halt disease progression rather than reverse changes, so it is becoming critical to identify $\mathrm{AD}$ early. Patients with AD typically present with symptoms caused by poor recall of recently learnt visual and verbal material; clinical testing reveals episodic memory deficits. ${ }^{23}$ Patients presenting with these symptoms and signs are diagnosed as having amnestic mild cognitive impairment (aMCI) if their problems are mild (with intact functional abilities) ${ }^{4}$ or probable $\mathrm{AD}$ if they have problems which impact on their lives. ${ }^{5}$ Many patients with aMCI progress to $\mathrm{AD}^{6}$ and patients with a diagnosis of aMCI or mild $\mathrm{AD}$ form a continuum, so we consider them as one group: aMCI/AD.
The diagnosis of cognitive disease requires a history from the patient and an informant, an examination including short cognitive tests and investigation. ${ }^{7}$ Short cognitive tests can be divided into three groups: orientation-dominated questionnaires, highly selective tests and multidomain tests. ${ }^{8}$ Multidomain tests are the most useful in aiding diagnosis. ${ }^{8}$ The most widely used short cognitive tests in memory clinics include the Addenbrooke's Cognitive Examinations (ACE-R). ${ }^{9} 10$

A clinical assessment including a multidomain cognitive test usually enables diagnosis in patients with moderate $\mathrm{AD}$. In milder cases, the diagnosis of $\mathrm{AD}$ is more difficult and may remain unclear after initial assessment. There are several methods available to clarify the diagnosis in mild cases including expert neuropsychological assessment, cerebrospinal fluid biomarkers, structural and functional brain imaging (including amyloid positron emission tomography). These are useful but share disadvantages: they require referral to specialists, take time and are expensive. This limits them to specialist services within highly developed countries. This study examines whether a targeted short cognitive test examining visual and verbal recall could be a cheap, effective method of supporting a diagnosis of $\mathrm{aMCI} / \mathrm{AD}$.

Some current multidomain short cognitive tests such as the ACEs,${ }^{910}$ Montreal Cognitive Assessment $(\mathrm{MOCA})^{11}$ and the Test Your Memory (TYM) $)^{12} 13$ are useful in patients with aMCI, but these tests are designed for several purposes and do not test visual recall (a cognitive skill affected early in $\mathrm{AD}$ ). The Test Your Memory for Mild Cognitive Impairment (TYM-MCI), formerly named the H-TYM, was designed to test verbal and visual recall to optimise its sensitivity to aMCI/AD. The TYM-MCI is shown in online supplementary appendix 1 .

In the validation study, the TYM-MCI was shown to be very powerful in distinguishing patients with mild AD from normal controls with excellent sensitivity (0.95) and specificity (0.93). ${ }^{14}$ This study examines the performance of the TYM-MCI in the more demanding and clinically relevant task of distinguishing patients with aMCI/AD from patients with subjective memory complaints (SMC) without neurological disease (for example, patients diagnosed as worried well or depressed).

\section{MATERIALS AND METHODS \\ Setting}

Patients were enrolled from a UK memory clinic between March 2011 and March 2014. A prospective cohort design was used to compare the 
TYM-MCI with the clinical diagnosis in patients supported as indicated by imaging, neuropsychological assessment and follow-up. Patients were recruited prospectively on a convenience basis-when nurses had time. The 96 patients with SMC and 58 patients with aMCI/AD recruited in this study provide $84 \%$ power to detect a difference of 2 points in the TYM-MCI score assuming a two-sided 5\% significance level and a SD of 4 based on a previous study. ${ }^{12}$

Patients were seen by a consultant neurologist and/or psychiatrist. All had a physical examination and completed the ACE-R, Mini-Mental State Examinations (MMSE), ${ }^{15}$ TYM test and TYM-MCI. The diagnoses of AD and aMCI were made according to current diagnostic criteria ${ }^{45}$ and were agreed at a multidisciplinary team (MDT) meeting; other diagnoses were made by consensus at the MDT. The specialist and the MDT had access to the ACE-R score but not the TYM or TYM-MCI. Follow-up and investigations were determined on clinical need at the MDT meeting; 57\% of the patients were followed up for between 4 months and 3 years. To select patients with mild problems, those scoring $\leq 24 / 30$ on the MMSE were excluded.

Ethical permission for the study was obtained from Cambridgeshire 2 Ethics Committee.

Patients were divided into six diagnostic groups:

1. SMC

2. $\mathrm{aMCI} / \mathrm{AD}$

3. No clear diagnosis

4. Fixed memory deficit head injury

5. Neurological disease sometimes associated with cognitive problems, for example, epilepsy

6. Other degenerative dementia

The analysis focuses on groups 1 and 2. Patients with mild non-amnestic cognitive problems were included in groups 3 to 5 rather than treated as a separate group.

\section{Administering and scoring the TYM-MCI}

The TYM-MCI was assessed in five ways:

1. As a single test in the diagnosis of aMCI/AD.

2. As a combined assessment with the ACE-R.

3. In patients with equivocal ACE-R scores.

4. Whether it adds value to the normal memory clinic assessment.

5. As a predictor of patients initially reassured but who are later rediagnosed with aMCI/AD.

The ordinary TYM test was also administered. To avoid confusion between the tests, the performance of the TYM test is in online supplementary appendix 2 .

The TYM-MCI was printed on both sides of a sheet of card (see online supplementary appendix 1).

The TYM-MCI was supervised by a nurse in a quiet room. Training the nurses took $5 \mathrm{~min}$. The patient was asked to copy the diagram and read the passage silently and then aloud. The patient answered the page 1 questions referring back to the passage. The patient was not told to memorise the answers. The card was turned over and the patient was asked to:

- reproduce the diagram within the square

- answer the same questions

- circle flowers mentioned in the passage

- answer new questions concerning the passage.

\section{Scoring}

Five page 2 recall tasks were scored:

1. Recall of the drawing $\quad / 15$

2. Recall of page one answers

3. Recognition of flowers from the passage

4. No flower misrecognition

5. Answering new questions on the passage

6. Total

The page two score=TYM-MCI score. Verbal recall 1 is the recall of answers already given, and verbal recall 2 is the new answers.

The cut-off of $\leq 13 / 30$ optimised in the validation study ${ }^{14}$ was used. The TYM-MCI, TYM and ACE-R were scored by the nurses with access to some clinical information.

\section{Statistical methods}

Cronbach's alpha was calculated for the TYM-MCI.

The distribution of the test scores did not follow a normal distribution so the Mann-Whitney U test was applied to compare between groups. Data from patients with SMC and aMCI/AD were used to plot a receiver operating characteristic (ROC) curve. Sensitivities, specificities and predictive values were calculated using standard methods. An 'or' rule was used for combined ROC analysis of the ACE-R and TYM-MCI. Correlations between the TYM-MCI and ACE-R were calculated using Spearman's r coefficient for non-parametric data.

To produce the age-matched groups, an age range of 51-80 years was set. The median value was obtained for each group, and all cases above the median were included for the SMC group and all cases below the median were included for the aMCI/AD group. Cases were then randomly sampled from the remaining cases to form equal sample sizes of 50 .

To determine whether the TYM-MCI provided additional information to standard tests, logistic regression was used to assess predictive ability. A logistic regression model was fitted to outcome (aMCI/AD or SMC) adjusting for age, MMSE and ACE-R. The integrated discriminant improvement ${ }^{16}$ was calculated to assess the improvement in model performance when the TYM-MCI is included compared with when it is excluded. Additional ROC curves were plotted using the predictive values generated from the models.

\begin{tabular}{lllllll}
\hline \multicolumn{6}{l}{ Table 1} & \multicolumn{6}{l}{ Characteristics and performance of the six groups on short cognitive tests including mean scores and SD } \\
\hline Group & Diagnosis & Patients (n) & Mean age & MMSE/30 & ACE-R/100 & TYM-MCI/30 \\
\hline 1 & SMC & 96 & 59.2 (SD 9.2) & 28.6 (SD 1.1) & 90.8 (SD 5.5) & 19.8 (SD 5.0) \\
2 & aMCI/AD & 58 & $67.8($ (SD 7.4) & 26.8 (SD 1.5) & 81.3 (SD 5.3) & 9.4 (SD 4.6) \\
3 & No clear diagnosis & 13 & $66.5($ SD 8.6) & 27.1 (SD 1.7) & 82.0 (SD 6.4) & 14.9 (SD 4.6) \\
4 & Fixed deficits & 11 & 61.7 (SD 8.8) & 27.6 (SD 1.5) & 84.0 (SD 6.0) & 13.4 (SD 7.4) \\
5 & Other neurological disease & 12 & 61.9 (SD 9.8) & 28.5 (SD 1.4) & 88.2 (SD 6.0) & 16.8 (SD 5.6) \\
6 & Non-AD dementia & 10 & 66.6 (SD 12.5) & 27.4 (SD 1.7) & 82.8 (SD 7.1) & 14.0 (SD 4.3) \\
\hline
\end{tabular}

ACE- $R$, Addenbrooke's Cognitive Examinations; aMCl/AD, amnestic mild cognitive impairment or Alzheimer's disease; MMSE, Mini-Mental State Examinations; SMC, subjective memory complaints; TYM-MCl, Test Your Memory for Mild Cognitive Impairment. 
Table 2 Comparison of the performance of the ACE-R, TYM-MCI subtests and TYM-MCI scores using effect size from a two-sample t-test and Mann-Whitney $U$ test $p$ value in patients with aMCI/AD and those with SMC

\begin{tabular}{|c|c|c|c|c|}
\hline & $\begin{array}{l}\text { Subjective memory complaints } \\
\text { (mean (SD)) }\end{array}$ & $\begin{array}{l}\text { Amnestic } \mathrm{MCl} / \mathrm{mild} \mathrm{AD} \text { (mean } \\
(\mathrm{SD}) \text { ) }\end{array}$ & Effect size* & $\mathrm{p}$ Value Mann-Whitney $\mathrm{U}$ test \\
\hline ACE-R & $90.8(5.5)$ & $81.3(5.3)$ & 11.0 & $<0.001$ \\
\hline Overall TYM-MCI & $19.8(5.0)$ & $9.4(4.6)$ & 13.0 & $<0.001$ \\
\hline Visual recall & $9.8(3.3)$ & $2.9(3.1)$ & 13.0 & $<0.001$ \\
\hline Verbal recall 1 & $4.4(0.9)$ & $3.1(1.1)$ & 8.5 & $<0.001$ \\
\hline Verbal recall 2 & $5.5(2.4)$ & $3.4(2.2)$ & 5.4 & $<0.001$ \\
\hline
\end{tabular}

* Mean difference divided by SE (effect size from a two sample t-test).

ACE-R, Addenbrooke's Cognitive Examinations; AD, Alzheimer's disease, aMCI/AD, amnestic mild cognitive impairment or Alzheimer's disease; TYM-MCl, Test Your Memory for Mild Cognitive Impairment.

\section{RESULTS}

\section{Patient characteristics}

Two hundred and two patients aged between 40 years and 80 years were enrolled. Two patients (one with SMC, one with aMCI) failed to complete the tests and were excluded. The characteristics of the remaining 200 are shown (table 1). There were no adverse events. Patients gave informed consent. The patients were divided into the six groups described, but principal analyses were restricted to the SMC and aMCI/AD groups in keeping with the primary aim of the study. The SMC patients formed the largest group with a mean age of 59.2 years, significantly lower than aMCI/AD patients (mean age 67.8 years). Therefore, additional analyses were performed on age-matched subsamples from the SMC and aMCI/groups.

\section{Reliability of the TYM-MCI}

Cronbach's alpha was 0.89 showing high internal consistency of the TYM-MCI.

The TYM-MCI and ACE-R as single tests to distinguish patients who have aMCI/AD from patients with SMC

Both the TYM-MCI and ACE-R detected a significant difference between patients with aMCI/AD and patients with SMC; the differences were proportionally larger in the TYM-MCI than the ACE-R (table 2). Effect size shows a proportionally larger difference for the visual recall task and the overall TYM-MCI

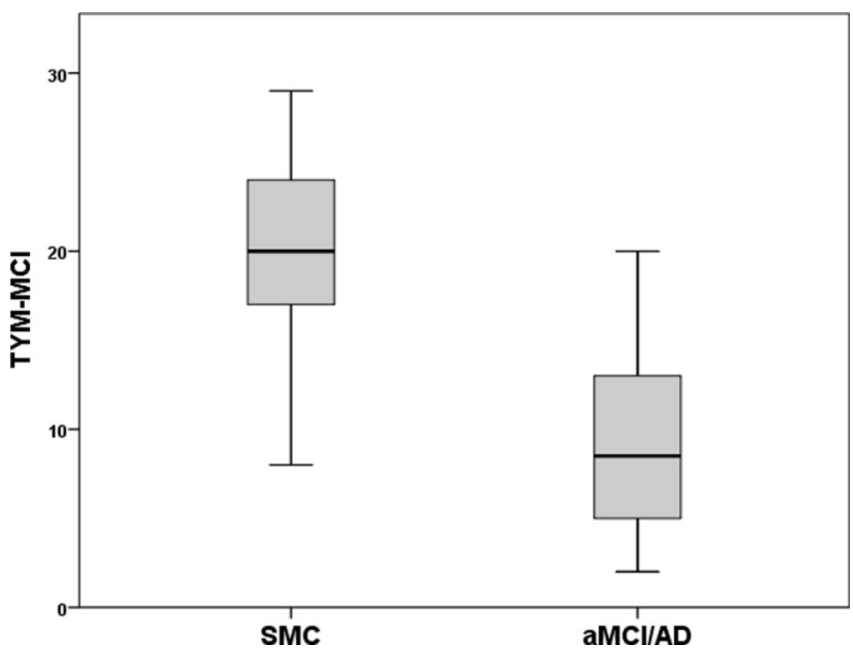

Figure 1 Box plot showing the distribution of the TYM-MCI score for patients with SMC and aMCI/AD. aMCI/AD, amnestic mild cognitive impairment or Alzheimer's disease; SMC, subjective memory complaints; TYM-MCl, Test Your Memory for Mild Cognitive Impairment. score compared with the ACE-R. Spearman's rho coefficient for non-parametric data was $0.66(\mathrm{p}<0.001)$ showing a moderately high correlation between the TYM-MCI and ACE-R.

The difference between patients with SMC and patients with aMCI/AD on the TYM-MCI is shown in figure 1 . There is a clear separation of most individuals: only patients with SMC scored $\geq 20$ and only patients with aMCI/AD scored $\leq 8$. The ROC curve (figure 2) compares the ability of the TYM-MCI, ACE-R and MMSE to distinguish patients with aMCI/AD from those with SMC. Sensitivities, specificities, positive predicative value (PPV) and negative predicative value (NPV) for the TYM-MCI and ACE-R are shown in table 3.

All tasks showed a significant difference between SMC patients and $\mathrm{aMCI} / \mathrm{AD}$ patients (table 2). The largest difference was for visual recall as in the original study. ${ }^{14}$

\section{Analysis of age-matched groups}

As there was a significant difference in age between the SMC and $\mathrm{aMCI} / \mathrm{AD}$ groups, further analyses were performed using age-matched cohorts (tables 4 and 5). Non-parametric MannWhitney $\mathrm{U}$ test showed no significant difference in the ages of the revised groups (SMC average age 64.4 years, aMCI/AD 66.7 years $\mathrm{p}=0.23$ ).

The area under the curve (AUC) of the ROC curve was 0.91 (95\% confidence limit (Cl) 0.86 to 0.96 ) for the TYM-MCI,

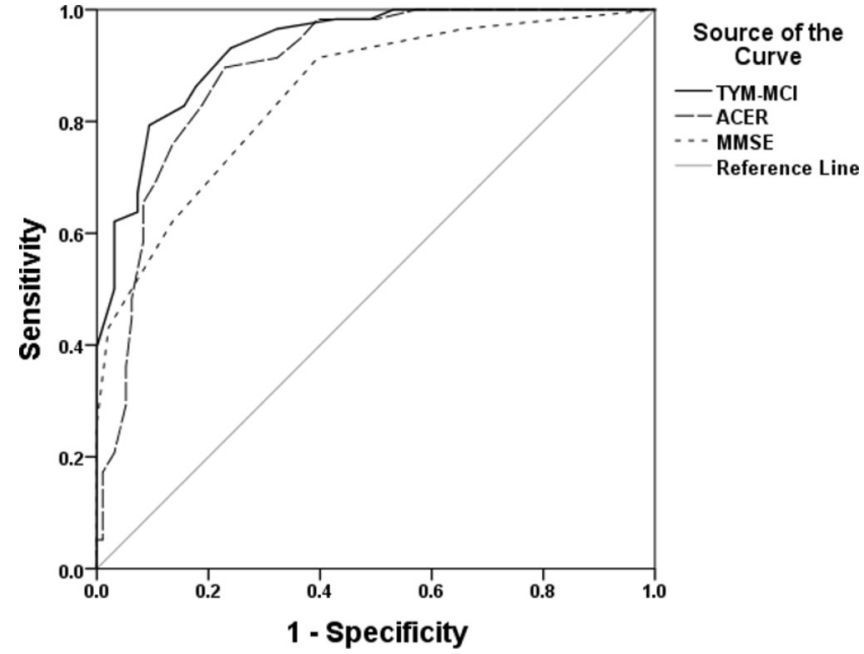

Figure 2 ROC curve for the TYM-MCl, ACE-R and MMSE in the separation of patients with aMCI/AD from those with SMC. ACE-R, Addenbrooke's Cognitive Examinations; aMCI/AD, amnestic mild cognitive impairment or Alzheimer's disease; MMSE, Mini-Mental State Examinations; TYM-MCl, Test Your Memory for Mild Cognitive Impairment. 
Table 3 Sensitivity and specificity of the ACE-R and TYM-MCI

\begin{tabular}{|c|c|c|c|c|c|c|c|}
\hline & Specificity & Sensitivity & $\begin{array}{l}\text { False negatives } \\
\text { (n) }\end{array}$ & False positives (n) & PPV & NPV & $\begin{array}{l}\text { AUC of continuous } \\
\text { score }\end{array}$ \\
\hline$A C E-R \leq 82$ & $0.92(0.84-0.96)$ & $0.59(0.45-0.71)$ & 24 & 8 & $0.81(0.66-0.91)$ & $0.79(0.70-0.86)$ & $0.89(0.84-0.94)$ \\
\hline ACE-R $\leq 87$ & $0.77(0.67-0.85)$ & $0.90(0.79-0.96)$ & 6 & 22 & $0.70(0.59-0.80)$ & $0.92(0.84-0.97)$ & $0.89(0.84-0.94)$ \\
\hline TYM-MCI $\leq 13$ & $0.91(0.83-0.96)$ & $0.79(0.67-0.89)$ & 12 & 9 & $0.84(0.71-0.92)$ & $0.88(0.80-0.94)$ & $0.93(0.89-0.97)$ \\
\hline
\end{tabular}

95\% confidence limits in brackets.

ACE-R, Addenbrooke's Cognitive Examinations; AUC, area under the curve; NPV, negative predicative value; PPV, positive predicative value; TYM-MCI, Test Your Memory for Mild Cognitive Impairment.

compared with 0.89 (95\% $\mathrm{Cl} 0.78$ to 0.96$)$ for the ACE-R. Using the cut-off of $\leq 13$, the sensitivity was $0.78(95 \% \mathrm{Cl} 0.65$ to 0.88$)$ and specificity $0.89(95 \% \mathrm{Cl} 0.78$ to 0.96$)$. This resulted in a PPV of $0.88(95 \% \mathrm{Cl} 0.75$ to 0.95$)$ and a NPV of $0.80(95 \%$ $\mathrm{Cl} 0.68$ to 0.89 ) for the TYM-MCI.

\section{Analysis of the TYM-MCl and ACE-R in combination}

The cross-classification of TYM-MCI and ACE-R scores against the clinical diagnosis is shown (table 6). Combining the results of the TYM-MCI with the ACE-R with an 'or' rule, the tests show a superior performance to either test alone. Using the criteria of scoring $\leq 82$ on the ACE-R or scoring $\leq 13$ on the TYM-MCI then the results are excellent with a sensitivity of $88 \%(95 \% \mathrm{Cl}$ $77 \%$ to $95 \%$ ) and specificity of $85 \%$ (95\% $\mathrm{Cl} 77 \%$ to $92 \%)$. Where it is crucial that no cases of aMCI/AD are missed then combining the ACE-R (cut-off 87) with the TYM-MCI yields a sensitivity of $97 \%$ (95\% Cl 88\% to $100 \%)$.

Analysis of TYM-MCl in patients with equivocal ACE-R scores The analysis on the 32 patients with equivocal ACE-R scores (83-87) showed that the TYM-MCI was very useful in this group. Mean score on the TYM-MCI was 17.2 for patients with SMC, 9.9 for patients with aMCI/AD. Eleven of fourteen patients with SMC and equivocal ACE-R scores passed the TYM-MCI. Thirteen of eighteen patients with aMCI/AD with equivocal ACE-R scores were detected by the TYM-MCI. The visual recall task was particularly useful (mean score 8.2 in patients with SMC, 3.2 in patients with aMCI/AD). The AUC of the ROC was 0.85 with a sensitivity of 0.72 and specificity of 0.79 (cut-off $\leq 13$ ). Using a cut-off of $\leq 18$, all cases of $\mathrm{aMCI} / \mathrm{AD}$ with equivocal ACE-R scores were detected by the TYM-MCI.
The TYM-MCl as an additional test to the ACE-R

Logistic regression analysis with integrated discriminant improvement analysis (table 7) showed that the TYM-MCI score is a highly significant predictor of the diagnosis of aMCI/AD $(p<0.0001)$ even after adjusting for the ACE-R score, MMSE and age. With model calibration, the logistic regression model which includes the TYM-MCI score confers an advantage over the model excluding the TYM-MCI: Nagelkerke's $\mathrm{R}^{2}$ statistic increased from 0.70 to 0.77 after including the TYM-MCI score and the Hosmer-Lemeshow $\chi^{2}$ statistic decreased from 8.02 $(p=0.43)$ to $5.52(p=0.70)$. ROC curves were plotted based on the model predictive values for each of the models. The AUC was 0.96 ( $\mathrm{Cl} 0.94$ to 0.99 ) for the model including the TYM-MCI score, compared with $0.93(95 \% \mathrm{Cl} 0.90$ to 0.97$)$ for the model without the TYM-MCI.

Integrated discriminant improvement (IDI) was used ${ }^{16}$ to quantify the significance of this difference using the new model including the TYM-MCI compared with the model without the TYM-MCI. The IDI was 0.10 (95\% confidence limits 0.05 to $0.16, \mathrm{p}<0.001$ ) showing that the TYM-MCI provides a significant improvement in model performance.

\section{ANALYSIS OF PATIENTS IN WHOM THE DIAGNOSIS WAS CHANGED TO AMCI/AD}

Thirteen patients diagnosed as SMC or unknown were subsequently rediagnosed with aMCI/AD (10 were under follow-up, 3 re-referred). These patients had significantly lower scores on their baseline TYM-MCI and ACE-R compared with all patients with SMC (table 8), they performed particularly poorly on visual recall.

\begin{tabular}{|c|c|c|c|c|}
\hline Short Cognitive Test or subtest & $\begin{array}{l}\text { Patients with subjective memory } \\
\text { complaints (mean (SD)) }\end{array}$ & $\begin{array}{l}\text { Patients with aMCI/AD (mean } \\
\text { (SD)) }\end{array}$ & Effect size* & $\begin{array}{l}\text { p Score Mann-Whitney } \\
\text { U test }\end{array}$ \\
\hline ACE-R & $91.1(5.4)$ & $81.4(5.5)$ & 9.4 & $p<0.001$ \\
\hline Total TYM-MCl & $19.3(5.2)$ & $9.5(4.7)$ & 10.3 & $p<0.001$ \\
\hline Visual recall & $9.4(3.3)$ & $3.1(3.2)$ & 10.3 & $\mathrm{p}<0.001$ \\
\hline Verbal recall 1 & $4.3(1.2)$ & $3.1(1.1)$ & 6.0 & $p<0.001$ \\
\hline Verbal recall 2 & $5.5(2.6)$ & $3.4(2.2)$ & 4.6 & $\mathrm{p}<0.001$ \\
\hline
\end{tabular}

* Mean difference divided by standard error (effect size from a two sample t-test).

ACE-R, Addenbrooke's Cognitive Examinations; aMCI/AD, amnestic mild cognitive impairment or Alzheimer's disease; TYM-MCl, Test Your Memory for Mild Cognitive Impairment.

Table 5 Sensitivity and specificity of the ACE-R and H-TYM in age-matched patients. 95\% confidence limits in brackets

\begin{tabular}{llllllll}
\hline & Specificity & Sensitivity & $\begin{array}{l}\text { False } \\
\text { negatives }(n)\end{array}$ & $\begin{array}{l}\text { False } \\
\text { positives }(n)\end{array}$ & PPV & NPV & $\begin{array}{l}\text { AUC of continuous } \\
\text { score }\end{array}$ \\
\hline ACE-R $\leq 82$ & $0.91(0.80$ to 0.97$)$ & $0.56(0.42$ to 0.70$)$ & 24 & 5 & $0.86(0.71$ to 0.95$)$ & $0.68(0.56$ to 0.78$)$ & $0.90(0.84$ to 0.96$)$ \\
ACE-R $\leq 87$ & $0.82(0.69$ to 0.91$)$ & $0.89(0.78$ to 0.96$)$ & 6 & 10 & $0.83(0.71$ to 0.92$)$ & $0.88(0.76$ to 0.96$)$ & $0.90(0.84$ to 0.96$)$ \\
TYM-MCI $\leq 13$ & $0.89(0.78$ to 0.96$)$ & $0.78(0.65$ to 0.88$)$ & 12 & 6 & $0.88(0.75$ to 0.95$)$ & $0.80(0.68$ to 0.89$)$ & $0.91(0.86$ to 0.96$)$ \\
\hline
\end{tabular}

ACE-R, Addenbrooke's Cognitive Examinations; NPV, negative predicative value; PPV, positive predicative value; TYM-MCl, Test Your Memory for Mild Cognitive Impairment. 
Table 6 Mean scores of the ACE-R cross-classified against the TYM$\mathrm{MCl}$ and the final diagnosis of subjective memory complaint

\begin{tabular}{|c|c|c|c|c|}
\hline & & \multicolumn{3}{|c|}{ TYM-MCI score } \\
\hline & & $0-13$ & $14+$ & Total \\
\hline \multicolumn{5}{|c|}{ (A) Patient with SMC } \\
\hline \multirow[t]{4}{*}{ ACE-R score } & $0-82$ & 3 & 5 & 8 \\
\hline & $83-87$ & 3 & 11 & 14 \\
\hline & $88+$ & 3 & 71 & 74 \\
\hline & Total & 9 & 87 & 96 \\
\hline \multicolumn{5}{|c|}{ (B) Patient with aMCI/AD } \\
\hline \multirow[t]{4}{*}{ ACE-R score } & $0-82$ & 29 & 5 & 34 \\
\hline & $83-87$ & 13 & 5 & 18 \\
\hline & $88+$ & 4 & 2 & 6 \\
\hline & Total & 46 & 12 & 58 \\
\hline
\end{tabular}

ACE-R, Addenbrooke's Cognitive Examinations; aMCI/AD, amnestic mild cognitive impairment or Alzheimer's disease; SMC, subjective memory complaint; TYM-MCI, Test Your Memory for Mild Cognitive Impairment.

Table 7 Logistic regression analysis to assess the predictive ability of the TYM-MCl, MMSE, ACE-R and age

\begin{tabular}{llll}
\hline & OR & $95 \%$ Cl & $\mathrm{p}$ Value \\
\hline TYM-MCl score & 0.75 & 0.65 to 0.86 & $\mathrm{p}<0.0001$ \\
MMSE & 0.82 & 0.47 to 0.97 & $\mathrm{p}=0.51$ \\
ACE-R & 0.85 & 0.75 to 0.97 & $\mathrm{p}=0.017$ \\
Age & 1.12 & 1.04 to 1.20 & $\mathrm{p}=0.002$ \\
\hline
\end{tabular}

ACE-R, Addenbrooke's Cognitive Examinations; MMSE, Mini-Mental State Examinations; TYM-MCI, Test Your Memory for Mild Cognitive Impairment.

\section{DISCUSSION}

The TYM-MCI is a targeted short cognitive test designed for a single purpose-the identification of aMCI/AD. The TYM-MCI is free, easy and quick to use and requires minimal training to administer. A previous study showed that the TYM-MCI was very powerful at distinguishing patients with aMCI/AD from healthy controls with an AUC of the ROC of $0.99 .{ }^{14}$ This study showed that the TYM-MCI performed very well on the useful and challenging task of distinguishing patients with aMCI/AD from those with SMC (with diagnoses such as worried well, depression, anxiety and functional problems) with an AUC of 0.93 .

A new cohort of patients attending a memory clinic who 'passed' the MMSE was recruited. The performance of the TYM-MCI in the diagnosis of aMCI or mild AD was assessed in comparison with and in combination with the ACE-R. The direct comparison of the tests needs to be interpreted with caution as there are two biases: first, the ACE-R and MMSE were available to the clinician whereas the TYM-MCI was not and second only patients who passed the MMSE were included. The first of these confounding factors may favour the ACE-R as the result will influence the specialist. The second precluded a direct comparison with the MMSE noting that it is well established that the MMSE is insensitive to aMCI/AD. ${ }^{12} 1718$ Patients were recruited in this study when there was sufficient time for nurses to administer tests, which could introduce a selection bias.

Clinicians were often uncertain of the diagnosis initially in this difficult group, but where the balance of evidence from clinical assessment, investigations and follow-up favoured aMCI/AD or SMC, this was recorded. The mean MMSE score of 26.8 in the aMCI/AD group was high, confirming that patients had mild disease. The TYM-MCI showed a complete separation of aMCI/ $\mathrm{AD}$ and SMC for patients with scores below 8 and above 20 . The ACE-R also performed well in this study. There were larger differences between the groups on the TYM-MCI compared with the ACE-R: the TYM-MCI scores of aMCI/AD patients were $53 \%$ of the TYM-MCI scores of SMC patients compared with a $10 \%$ difference in the ACE-R score. The largest difference was in visual recall. Calculation of the effect size showed better performance of the TYM-MCI and the visual recall subtest compared with the ACE-R in distinguishing aMCI/AD from SMC.

Neuropsychological profiles of AD show that the characteristic earliest features of mild $\mathrm{AD}$ are poor recall of recently learnt visual and verbal material and these can be present and appear stable for years in the 'pre-dementia' stage of AD pathology. ${ }^{19}$ Although two short tests that are used occasionally test visual recall, the Mini-Mental Parkinson's ${ }^{20}$ and the visual association test ${ }^{21}$ visual recall is often ignored in the clinical assessment of suspected AD. The TYM-MCI is the first short cognitive test to test both visual and verbal recall making it a suitable instrument to detect disease in this important group of patients.

Additional analyses performed with age-matched sub-groups from patients with $\mathrm{aMCI} / \mathrm{AD}$ and SMC showed that the differences remained highly significant for all the TYM-MCI subtests with similar effect sizes, PPV and ROC curve. Logistic regression analysis confirmed that the TYM-MCI result remained significant after adjusting for age.

Addition of the TYM-MCI result to the ACE-R (cut-off 87) improves its performance: only 2 of the $58 \mathrm{aMCI} / \mathrm{AD}$ patients passed both tests and only 6 of the 96 SMC patients failed both tests.

Examining the usefulness of the TYM-MCI in the 32 patients with equivocal ACE-R scores showed it performed very well with a high AUC, sensitivity and specificity in distinguishing such patients with aMCI/AD from those with SMC. Logistic regression analysis showed that the TYM-MCI score was a highly significant predictor of aMCI/AD $(p<0.0001)$ even after adjusting for ACE-R, MMSE scores and age. The integrated discriminant improvement showed that using the TYM-MCI

Table 8 Mean baseline scores for the patients whose diagnosis changed to aMCI/AD compared with patients in group 1 (SMC)

\begin{tabular}{lllll}
\hline $\begin{array}{l}\text { Short Cognitive Test or } \\
\text { subtest }\end{array}$ & $\begin{array}{l}\text { Mean scores of patients whose diagnosis } \\
\text { changed to aMCI/AD }(\mathbf{n}=13)\end{array}$ & $\begin{array}{l}\text { Subjective memory } \\
\text { complaints }(\mathbf{n}=96)\end{array}$ & Effect size* & $\begin{array}{l}\mathrm{p} \text { Score Mann-Whitney } \\
\text { U test }\end{array}$ \\
\hline ACE-R & $83.5(4.4)$ & $90.8(5.5)$ & 4.6 & $\mathrm{p}<0.001$ \\
\hline Total TYM-MCI & $9.8(4.7)$ & $19.8(5.0)$ & 6.9 & $\mathrm{p}<0.001$ \\
Visual recall & $3.4(2.9)$ & $9.8(3.3)$ & 6.6 & $\mathrm{p}<0.001$ \\
\hline Verbal recall 1 & $2.7(1.3)$ & $4.4(0.9)$ & 6.2 & $\mathrm{p}<0.001$ \\
Verbal recall 2 & $3.7(2.2)$ & $5.5(2.4)$ & 2.5 & $\mathrm{p}=0.02$ \\
\hline
\end{tabular}

* Mean difference divided by standard error (effect size from a two sample t-test).

ACE-R, Addenbrooke's Cognitive Examinations; aMCI/AD, amnestic mild cognitive impairment or Alzheimer's disease; SMC, subjective memory complaint; TYM-MCl, Test Your Memory for Mild Cognitive Impairment. 
provides a significant improvement in model performance compared with a model that used age, MMSE and ACE-R only.

In this study, the TYM-MCI has a sensitivity of 0.79 . Therefore, used alone it will not detect some cases of aMCI/AD. Using the TYM-MCI with the ACE-R improves the sensitivity or increasing the TYM-MCI cut-off to $16 / 30$ increases the sensitivity to 0.93 .

Patients diagnosed with SMC are a heterogeneous group including patients with no organic disease, psychiatric problems or other diseases. A few patients with an original diagnosis of SMC later present with aMCI/AD: 13 patients in this study who were initially felt not to have aMCI/AD were later diagnosed with it. These 13 individuals scored significantly lower than other SMC patients on all subtests of the TYM-MCI (particularly visual recall). A low score on the TYM-MCI cautions against diagnosing SMC.

The scores on the TYM-MCI in the most difficult patientsthose with equivocal ACE-R scores and patients whose diagnosis changed were similar to those of the whole aMCI/AD cohort. This suggests that the TYM-MCI score may fall early in the disease and then plateau. This corresponds with physiological and anatomic studies which show severe involvement of hippocampal and parahippocampal structures early in the disease prior to AD pathology spreading to other areas. ${ }^{19}$ This suggests the TYM-MCI may detect aMCI/AD prior to the development of a generalised dementia.

Another study has examined the use of the TYM-MCI in a memory clinic. ${ }^{22}$ The TYM-MCI was administered to 38 patients in whom the diagnosis of MCI or subjective memory impairment was uncertain. With a cut-off of $15 / 30$, the TYM-MCI proved very sensitive for detecting MCI when the diagnosis was unclear, the author concluded that the TYM-MCI was useful in the assessment of such patients.

The current study has several limitations: the recruitment period of this study overlapped with an earlier study. ${ }^{14}$ The two studies had different inclusion criteria but patients with aMCI/ $\mathrm{AD}$ could be recruited into either. There was no dual recruitment but not all patients meeting criteria were recruited into this study. Based on a comparison of results from the studies if all aMCI/AD patients had been included in this study it would have increased the number of patients in the aMCI/AD group and lowered the TYM-MCI scores of the aMCI/AD cohort further increasing the power and observed effect sizes. Nonetheless, this study was adequately powered.

The interpretation of cognitive tests depends on the clinical setting. This study was performed in a NHS clinic and patients were managed by experienced memory specialists according to their usual practice. This makes the results applicable to similar settings but there was some variation in investigative practice. The patients were diagnosed clinically with support from neuroimaging, neuropsychological assessment and follow-up in selected individuals. Use of cerebrospinal fluid (CSF) biomarkers and more specialised functional and structural imaging could support the clinical diagnosis but were not practical to use in all subjects. The diagnosis of aMCI/AD was based on international consensus criteria rather than biomarkers.

In summary, the TYM-MCI performs at least as well as the ACE-R as a cognitive test in distinguishing patients with mild aMCI/AD from those with SMC. Further studies are needed to directly compare the TYM-MCI with other short cognitive tests such as the MOCA ${ }^{11}$ and Qmci. ${ }^{23}$ The TYM-MCI is useful in the diagnosis aMCI/AD in patients with equivocal ACE-R scores and in aMCI/AD patients initially diagnosed as having SMC or unknown. Uniquely among short cognitive tests the TYM-MCI examines visual and verbal recall. The TYM-MCI shows a consistent pattern with very low scores on visual recall and low scores on verbal recall even in the mildest patients. It adds value to the ACE-R. Very few patients with aMCI/AD pass both tests and very few patients with SMC fail both tests. Importantly for clinicians the TYM-MCI is free, quick and easy to use. It takes 5 min to learn how to administer the TYM-MCI. The TYM-MCI is a very useful aid in the diagnosis of aMCI/AD, alone or in combination with the ACE-R. The TYM-MCI will be available to download free from the website tymtest.com.

Contributors $\mathrm{JMB}$ is the guarantor of this study. He designed the study and wrote the initial draft of the manuscript. JW, KED and KH administered the TYM-MCI to patients and scored the results. CJL and RAP performed the statistical analysis and helped to write the text. JBR contributed to the planning of the study and in writing the text.

Funding JBR was supported by the Wellcome Trust (103838).

Competing interests None declared.

Ethics approval Cambridgeshire 2 .

Provenance and peer review Not commissioned; externally peer reviewed.

Open Access This is an Open Access article distributed in accordance with the terms of the Creative Commons Attribution (CC BY 4.0) license, which permits others to distribute, remix, adapt and build upon this work, for commercial use, provided the original work is properly cited. See: http://creativecommons.org/ licenses/by/4.0/

(C) Article author(s) (or their employer(s) unless otherwise stated in the text of the article) 2017. All rights reserved. No commercial use is permitted unless otherwise expressly granted.

\section{REFERENCES}

1 Ferri CP, Prince M, Brayne C, et al. Global prevalence of dementia: a Delphi consensus study. Lancet 2005;366:2112-7.

2 Greene JD, Baddeley AD, Hodges JR. Analysis of the episodic memory deficit in early Alzheimer's disease: evidence from the doors and people test. Neuropsychologia 1996;34:537-51

3 Nestor P, Scheltens P, Hodges J. Advances in the early detection of Alzheimer's Disease. Nat Rev Neurosci 2004;5:S34-S41.

4 Albert MS, DeKosky ST, Dickson D, et al. The diagnosis of mild cognitive impairment due to alzheimer's disease: recommendations from the National Institute on AgingAlzheimer's Association workgroups on diagnostic guidelines for Alzheimer's disease. Alzheimers Dement 2011;7:270-9.

5 McKhann GM, Knopman DS, Chertkow H, et al. The diagnosis of dementia due to alzheimer's disease: recommendations from the National Institute on AgingAlzheimer's Association workgroups on diagnostic guidelines for Alzheimer's disease. Alzheimers Dement 2011;7:263-9.

6 Dubois B, Albert ML. Amnestic MCl or prodromal alzheimer's disease? Lancet Neurol 2004;3:246-8

7 London; National Institute for Health and Clinical Excellence. Dementia: supporting people with dementia and their Carers in Health and Social Care. NICE technology guideline number 2006;42.

8 Brown J. The use and misuse of short cognitive tests in the diagnosis of dementia. J Neurol Neurosurg Psychiatry 2015;86:680-5.

9 Mathuranath PS, Nestor PJ, Berrios GE, et al. A brief cognitive test battery to differentiate Alzheimer's disease and frontotemporal dementia. Neurology 2000;55:1613-20.

10 Mioshi E, Dawson K, Mitchell J, et al. The Addenbrooke's Cognitive Examination Revised (ACE-R): a brief cognitive test battery for dementia screening. Int J Geriatr Psychiatry 2006;21:1078-85.

11 Nasreddine ZS, Phillips NA, Bédirian V, et al. The Montreal Cognitive Assessment, MoCA: a brief screening tool for mild cognitive impairment. J Am Geriatr Soc 2005;53:695-9.

12 Brown J, Pengas G, Dawson K, et al. Self administered screening test (TYM) for the detection of AD: a cross sectional study. BMJ 2009;338:b203.

13 Hanyu $H$, Maezono M, Sakurai $H$, et al. Japanese version of the test your memory as a screening test in a japanese memory clinic. Psychiatry Res 2011;190:145-8.

14 Brown JM, Wiggins J, Dong $\mathrm{H}$, et al. The hard test your memory. evaluation of a short cognitive test to detect mild Alzheimer's disease and amnestic mild cognitive impairment. Int J Geriatr Psychiatry 2014:29:272-80.

15 Folstein MF, Folstein SE, McHugh PR. "Mini-mental state". A practical method for grading the cognitive state of patients for the clinician. J Psychiatr Res 1975;12:189-98

16 Pencina MJ, D'Agostino RB, D'Agostino RB, et al. Evaluating the added predictive ability of a new marker: from area under the ROC curve to reclassification and beyond. Stat Med 2008;27:157-72. 
17 Galasko D, Klauber MR, Hofstetter CR, et al. The Mini-Mental state examination in the early diagnosis of Alzheimer's disease. Arch Neurol 1990;47:49-52.

18 Mitchell AJ. The Mini-Mental State Examination (MMSE): update on its diagnostic accuracy and clinical utility for cognitive disorders. In: Larner AJ, ed. CognitiveScreening Disorders. A Practical Approach. $2^{\text {nd }}$ edition. London: Springer 2017:37-48.

19 Bäckman L, Small BJ, Fratiglioni L. Stability of the preclinical episodic memory deficit in Alzheimer's disease. Brain 2001;124:96-102.
20 Mahieux F, Michelet D, Manifacier MJ, et al. Mini-Mental Parkinson: first validation study of a new bedside test constructed for Parkinson's disease. Behav Neurol 1995;8:15-22

21 Lindeboom J, Schmand B, Tulner L, et al. Visual association test to detect early dementia of the alzheimer type. J Neurol Neurosurg Psychiatry 2002;73:126-33.

22 Larner AJ. Hard-TYM: a pragmatic study. Int I Geriatr Psychiatry 2015;30:330-1.

23 O'Caoimh R, Gao Y, McGlade C, et al. Comparison of the quick mild cognitive impairment (Qmci) screen and the SMMSE in screening for mild cognitive impairment. Age Ageing 2012;41:624-9. 